\title{
Desempenho bioeconômico de sistemas intensivos de cria e de ciclo completo por meio de simulação
}

\author{
[Bioeconomic performance of intensive breeding systems and of \\ full-cycle by way of simulation] \\ R.A.V. Santana ${ }^{1}$, F.A. Barbosa ${ }^{2}$, R.A. Mandarino ${ }^{3}$, C.F. Lobo $^{4}$ \\ ${ }^{1}$ IFNMG - Arinos, MG \\ ${ }^{2}$ Escola de Veterinária - Universidade Federal de Minas Gerais - Belo Horizonte, MG \\ ${ }^{3}$ Aluno de pós-graduação - Escola de Veterinária - Universidade Federal de Minas Gerais - Belo Horizonte, MG \\ ${ }^{4}$ Aluno de pós-graduação - FAV - Universidade de Brasília - Brasília, DF
}

\begin{abstract}
RESUMO
Foram simulados quatro sistemas de produção de bovinos de corte a partir de dados zootécnicos e econômicos de uma fazenda de ciclo completo situada no município de Corinto, região central de Minas Gerais. Utilizou-se o programa de simulação bioeconômica Embrapec. Os sistemas simulados foram: ciclo completo com uso de cruzamento industrial (CompCRUZA), ciclo completo exclusivamente com zebuíno (CompZEBU), ciclo de cria com uso de cruzamento industrial (CriaCRUZA), ciclo de cria exclusivamente com zebuíno (CriaZEBU). No sistema com cruzamento industrial, os animais eram vendidos logo após a desmama, com oito meses (CriaCRUZA), ou abatidos aos 13 meses (CompCRUZA). No sistema com rebanho zebu, os animais eram também vendidos à desmama (CriaZEBU) ou abatidos aos 24 meses (CompZEBU). As bezerras foram recriadas, e uma parcela (15\%), descartada para a venda. A quantidade de animais em um rebanho estabilizado foi $13,1 \%$ e $10,7 \%$ menor nos sistemas cruzados, completo e cria, respectivamente; porém a quantidade $(\mathrm{kg})$ de carcaça vendida/ha (49,9 para CriaCRUZA e 118,1 para CompCRUZA) e a taxa de desfrute $(24,4 \%)$ foram em média $15,3 \%$ maior que zebuínos. A taxa interna de retorno e o valor presente líquido foram superiores para os sistemas que adotaram o cruzamento de zebuínos com raças europeias, CriaCRUZA $(17,2 \%$ e R $\$ 10.151 .896,54)$ e CompCRUZA $(18,9 \%$ e R $\$ 11.749 .329,42)$. Quando comparados os sistemas de produção, os que utilizaram ciclo completo, o CompCRUZA $(18,9 \%$ e $\mathrm{R} \$ 11.749 .329,42)$ e CompZEBU $(16,2 \%$ e $\mathrm{R} \$$ 9.568.293,51), foram em média 18,7\% maiores que os de cria, quando comparados com o mesmo grupo genético, CriaCRUZA $(17,2 \%$ e $\mathrm{R} \$ 10.151 .896,54)$ e CriaZEBU $(14,6 \%$ e $\mathrm{R} \$ 7.955 .230,38)$. Todos os sistemas de produção simulados foram economicamente viáveis, sendo que o sistema de produção CompCRUZA foi que apresentou maior viabilidade econômica e com melhores indicadores zootécnicos.
\end{abstract}

Palavras-chave: cruzamento industrial, gado de corte, indicadores zootécnicos, rentabilidade, zebu

\begin{abstract}
Four alternative systems of production from zootechnical and economic beef cattle data were simulated from an existing full-cycle farm located in Corinto, state of Minas Gerais, Brazil. The systems simulated were: full-cycle with the use of crossbreed cattle (CompCRUZA), full cycle, exclusively with zebu cattle (CompZEBU), rearing cycle with the use of crossbreed cattle (CriaCRUZA) and rearing cycle with zebu cattle (CriaZEBU). The crossbreeding system animals were sold immediately after weaning with 8 months (CriaCRUZA) or slaughtered with 13 months (CompCRUZA). The zebu cattle system animals were also sold after weaning (CriaZEBU) or slaughtered with 24 months (CompZEBU). The female calves were reared and $15 \%$ were destined for sale. The data was simulated on the bioeconomic software "Embrapec". The number of animals in the stabilized herd was lower in cross-systems, but the amount $(\mathrm{kg})$ of carcass sold/ha (49.9 for CriaCRUZA and 118.1 for CompCRUZA) and offtake rate (24.4\%) were
\end{abstract}

Recebido em 26 de março de 2012

Aceito em 13 de junho de 2013

E-mail: ronan.valadares@ifnmg.edu.br 
superior to zebu cattle. The internal rate of return and net present value was higher for systems that have adopted the crossing system (taurine $x$ zebu cattle), CriaCRUZA $(17.2 \%$ and $R \$ 10,151,896.54)$ and CompCRUZA (18.9\% and $R \$ 11,749,329.42)$. When comparing the production systems, those using fullcycle, the CompCRUZA and CompZEBU (16.2\% and $R \$$ 9,568,293.51), were more profitable than rearing systems, when compared with the same genetic group, CriaCRUZA and CriaZEBU (14.6\% to R\$ 7,955,230.38. All production systems simulated were financially feasible, but the production systemCompCRUZA showed the highest economic viability and better zootechnical indicators.

Keywords: crossing, beef cattle, zootechnical indicators, profitability, zebu

\section{INTRODUÇÃO}

A profissionalização da cadeia produtiva agropecuária vem sendo forçada pela contínua diminuição da margem de lucro dos produtos agropecuários.

Cada vez mais exige-se o aumento do investimento com o objetivo de ampliar a escala de produção, aumentando a competitividade e diminuindo os custos unitários, principalmente o fixo. Onde, quando, como e quanto investir são, muitas vezes, indagações difíceis de responder, pois envolvem uma série de fatores administrativos, econômicos e culturais.

A pecuária intensiva de corte, de cria e ciclo completo, é caracterizada pelo maior uso de insumos em todas as etapas de produção, em comparação com o sistema extensivo. O nível de intensificação é diretamente proporcional à quantidade utilizada desses insumos. Quanto maior o uso de fertilizantes em pastagem ou de suplementos alimentares, por exemplo, maior será a velocidade de rebrota, resultando em capacidade de suporte mais elevada ou maior o ganho de peso dos animais, respectivamente.

A intensificação da produção pecuária provoca redistribuição de recursos dentro da estrutura organizacional da empresa. Esse processo desenvolve-se no incremento dos planos administrativo, sanitário, genético, alimentar e de técnicas de manejo do rebanho. Entretanto, embora a pesquisa seja abundante quanto às tecnologias biológicas para melhoria dos indicadores produtivos, existe deficiência de trabalhos que integrem o nível de evolução do sistema (e suas multivariáveis: ambiente, animais e mercado) com sua real eficiência bioeconômica (Tanure \& Nabinger, 2010).

A complexidade das relações existentes entre os fatores que compõem o sistema pecuário dificulta a avaliação do impacto de estratégias na produtividade, e, por consequência, na tomada de decisão pelo produtor. As ferramentas tradicionais utilizadas para a tomada de decisões têm sido cada vez mais questionadas. As variáveis custo e tempo têm sido apontadas como os principais problemas para a solução dos entraves gerenciais das empresas rurais (Ferreira et al., 2002).

Com o avanço das pesquisas, cada vez mais novas tecnologias se tornam disponíveis aos produtores. Os modelos bioeconômicos podem auxiliar na implantação ou na substituição de tecnologias. O uso de modelos matemáticos que unificam os parâmetros biológicos (produção) e econômicos, quando bem aplicados, são uma importante ferramenta de tomada de decisão. Assim, novos caminhos podem ser escolhidos com relativa segurança, evitando prejuízos tanto financeiros quanto sociais e ambientais.

O objetivo deste trabalho foi realizar uma avaliação "ex-ante" para comparar o desempenho bioeconômico de sistemas intensivos de cria e de ciclo completo de bovinos de corte por meio de simulação.

\section{MATERIAL E MÉTODOS}

A pesquisa foi realizada na Fazenda Cristal, localizada em Corinto, na região central do Estado de Minas Gerais. A propriedade está a uma altitude média de 587,5 metros e tem como coordenadas geográficas UTM, 548.461, 7.985.501 23k (Datum WGS84). A área total é de 5.071,1 hectares (ha), sendo que 1.267,8ha destes são destinados à preservação ambiental. A área destinada à pecuária de corte é composta totalmente de pastagens de origem antrópica (cultivadas), correspondendo a 3.803,3ha, distribuídos em 3.753,3ha com as espécies: Panicum maximun cv. Tanzania I, Brachiaria brizantha cv. Marandu e Xaraés, Brachiaria 
decumbens e Andropogon Gayanus, em sequeiro e 50ha da espécie Brachiaria brizantha cv. Marandu irrigados via pivô central. O volumoso utilizado no confinamento é a cana-de-açúcar (Saccharum officinarum), que ocupa uma área de 37ha.

Os parâmetros zootécnicos e a estrutura de produção, utilizados na simulação dos sistemas de produção, foram levantados na propriedade em estudo e representaram a média de cinco anos. Foram considerados os valores reais da fazenda para compra de insumos, bem como das categorias animais, arroba do boi gordo e vaca e venda de bezerros. Os dados econômicos usados foram coletados no período de janeiro a dezembro de 2011. A capacidade de suporte das pastagens de sequeiro foi de 1,2 UA/ha nas águas e $0,9 \mathrm{UA} / \mathrm{ha}$ na seca, e nas pastagens irrigadas foi de 6,0 e 3,0 UA/ha nas águas e na seca, respectivamente, sendo a mesma para todos os sistemas simulados. As adubações de manutenção foram feitas de duas formas: parceladas ao longo de cada ano na área de pastagem irrigada e uma vez a cada quatro anos nas demais áreas de pastagem em sequeiro. $\mathrm{O}$ custo da área de pastejo intensivo foi de $\mathrm{R} \$$ 2.060,20/ha/ano e da semi-intensiva $\mathrm{R} \$$ 135,50/ha/ano.

A estação de monta foi de janeiro a março, sendo usada a inseminação artificial em tempo fixo (IATF) no primeiro serviço e repasse com touro zebuíno (Tabapuã, Nelore e Brahman). As novilhas cruzadas com as raças Angus (geração F1) e Brangus (geração F2) entram na reprodução aos 14 meses de idade, e as novilhas zebuínas, aos 26 meses de idade. As novilhas tricross (1/2 NelorexAngus $\mathrm{X}$ 1/2Charolês, 1/2 TabapuãxAngus X 1/2Charolês, $1 / 2$ NelorexAngus X 1/2Brahman, $1 / 2$ TabapuãxAngus X 1/2Brahman, 3/4 Nelore x 1/4 Angus, 3/4 Tabapuã $\mathrm{x} \quad 1 / 4$ Angus) foram terminadas em confinamento e abatidas aos 13 meses de idade como superprecoce, com $485 \mathrm{~kg}$. Os animais zebuínos foram abatidos aos 24 meses de idade em confinamento, com $485 \mathrm{~kg}$.

Foram simulados quatro sistemas intensivos de produção: ciclo completo com uso de cruzamento industrial (CompCRUZA), ciclo completo exclusivamente com zebuíno (CompZEBU), ciclo de cria com uso de cruzamento industrial (CriaCRUZA), ciclo de cria exclusivamente com zebuíno (CriaZEBU). No sistema com cruzamento industrial, os animais eram vendidos logo após a desmama, com 8 meses (CriaCRUZA), ou abatidos, produzindo os novilhos superprecoces (CompCRUZA). No sistema com rebanho zebu, os animais eram também vendidos à desmama, com 8 meses (CriaZEBU), ou abatidos como novilhos precoces com 24 meses (CompZEBU). Os bezerros (machos) eram vendidos no mês de maio de cada ano. As bezerras desmamadas foram mantidas no rebanho até completarem um ano, quando da seleção e descarte de $15 \%$ delas. As novilhas descartadas foram vendidas em julho de cada ano. As novilhas de reposição tinham o primeiro parto após os dois anos de idade, no sistema cruzado, e aos três, no zebuíno. As vacas velhas foram descartadas aos seis anos de idade, sempre no mês de junho de cada ano, bem como as vazias, independentemente da idade. Para a simulação, a estação de monta ocorreu em janeiro, e os nascimentos, em outubro. As taxas de natalidade foram de $85 \%$, com exceção das primíparas, que foi de $65 \%$. O rebanho inicial foi determinado no levantamento dos dados e fornecido ao modelo por intermédio do número de animais em cada categoria. Por se tratar de um rebanho composto por animais zebuínos e cruzados com europeus, admitiu-se o mesmo número de animais para cada sistema. A estabilização do rebanho foi feita pelo simulador, utilizando a taxa de lotação nos períodos das águas e de seca, as taxas de mortalidade das diferentes categorias, taxas de descarte para vacas, novilhas e touros, taxa de natalidade e idade à primeira parição, todas retiradas da média obtida no rebanho nos últimos cinco anos. As taxas de mortalidade consideradas foram de $1,5 \%, 1 \%$ e $0,5 \%$, para até a desmama, da desmama aos dois anos de idade, e acima de 3 anos e touros, respectivamente.

O manejo nutricional existente na fazenda foi mantido nas simulações. Todos os sistemas simulados utilizaram as mesmas áreas de pastagens com a mesma capacidade de suporte e proporção de sequeiro e irrigadas descritas anteriormente. Nos sistemas com animais cruzados, foi utilizada a suplementação com "creepfeeding" para machos e fêmeas. Após a desmama, realizada em maio de cada ano, os bezerros eram levados para o confinamento, no período de junho a novembro (CompCRUZA), ou vendidos (CriaCRUZA). As fêmeas 
eram suplementadas com suplemento proteico-energético, e o ganho de peso foi de $200 \mathrm{~g} /$ dia. No sistema CompZEBU não foi utilizado "creepfeeding", porém houve uma suplementação na primeira seca, com suplemento proteico-energético para machos e fêmeas, durante os meses de junho a outubro de cada ano. $\mathrm{O}$ ganho de peso considerado no período chuvoso foi de $600 \mathrm{~g} / \mathrm{dia}$, e $200 \mathrm{~g} / \mathrm{dia}$ no período seco. Na segunda seca, os machos eram confinados nos meses de agosto a outubro. O suplemento mineral era específico para cada categoria no período das águas. No período seco, as matrizes eram suplementadas com mineral e ureia.

$\mathrm{O}$ custo do confinamento ( $\mathrm{R} \$ 3,50 /$ cab.dia) considerou apenas a alimentação, uma vez que o programa calcula os outros custos separadamente. A dieta foi formulada para ganho de $1,5 \mathrm{~kg} / \mathrm{dia}$, sendo a mesma já utilizada no confinamento da fazenda, tanto para os animais do sistema CompCRUZA quanto para os do CompZEBU.

Os preços dos bezerros desmamados de zebuínos e de cruzamento industrial e das demais categorias foram levantados de acordo com as últimas vendas e/ou compras realizadas pela fazenda em leilões e para outros criadores durante o ano de 2011. Os valores da arroba de boi gordo ( $R \$ 100,00)$, da vaca gorda $R \$(90,00)$, de bezerros desmamados nelore $(\mathrm{R} \$ 740,00)$ e cruzados ( $\mathrm{R} \$$ 800,00) foram baseados nas últimas vendas aos frigoríficos e em leilões no ano de 2011.

Os custos com medicamentos diversos, com carrapaticidas e com a inseminação artificial em tempo fixo não são computados pelo modelo e foram incluídos como gastos diversos.

Para a avaliação da viabilidade econômica, foi feita uma análise baseada na metodologia proposta por Frank (1978) e utilizada por Costa et al. (1986), Corrêa et al. (2000), Guimarães (2006) e Barbosa et al. (2010). Foram usados os indicadores de margem bruta (receitas totais custos variáveis); lucro operacional (receitas totais - (custos variáveis + depreciações)); lucro total (lucro operacional - juros). Para o cálculo dos juros, foi usada a metodologia de valores decrescentes em que as taxas de juros devem ser maiores para o capital circulante do que para o capital fundiário, e intermediárias para o capital de exploração fixa, sendo que a taxa de juros máxima adotada será de $6,75 \%$ ao ano (equivalente aos juros para custeio pecuário de financiamento). Foi utilizado também o valor presente líquido (VPL), que é definido como a diferença entre o valor presente dos fluxos de caixa futuros, descontados à taxa mínima de atratividade (TMA) e a taxa interna de retorno (TIR).A TIR de um fluxo de caixa é dada como a taxa de desconto que torna seu VPL igual a zero. Sua principal facilidade de uso é que o resultado é uma taxa de juros, bastando compará-la à TMA (Carvalho et al., 2009).

A estrutura inicial do rebanho foi fornecida ao modelo por intermédio do número de animais em cada categoria. $\mathrm{O}$ crescimento ou estabilização e o desempenho do rebanho foram controlados pelos parâmetros zootécnicos adotados.

$\mathrm{O}$ preço da terra foi o valor médio da região, $\mathrm{R} \$$ $2.920,00 /$ ha. Os valores das benfeitorias existentes na Fazenda Cristal somaram $\mathrm{R} \$$ 1.846.336,00, e nos sistemas de cria foram descontados R \$ 241.974,00 referentes à estrutura de confinamento. As máquinas e equipamentos somaram R \$ 597.519,00, considerando também outros equipamentos existentes, como computadores, impressoras, celulares, rádios, etc. A vida útil considerada foi de $25,20,20$ e 10 anos para edificações, currais, cercas e máquinas e equipamentos, respectivamente, sendo o valor residual de $20 \%$. Os veículos tiveram uma vida útil de 5 anos e valor residual de $40 \%$. As manutenções de construções e veículos foram de $3 \%$ e $5 \%$, respectivamente, ficando classificadas como custos fixos. Os investimentos, custos e receitas do fluxo de caixa e os indicadores de eficiência econômica e biológica dos sistemas propostos foram analisados em um horizonte de 10 anos, e os dados foram processados em um simulador bioeconômico. O programa utilizado foi o Embrapec, versão 1.0, que é um programa de simulação de sistemas bioeconômicos, desenvolvido no Centro Nacional de Pesquisa de Gado de Corte - Embrapa (Cezar, 1982).

\section{RESULTADOS E DISCUSSÃO}

O número médio de cabeças existentes no mês de outubro dos dez anos projetados e respectivo desvio padrão está na Tabela 1 . 
Desempenho bioeconômico...

Tabela 1. Composição média do rebanho nos meses de outubro dos dez anos de projeção (número de cabeças e desvio padrão (DP) de acordo com os sistemas simulados, CriaCRUZA, CompCRUZA, CriaZEBU, CompZEBU

\begin{tabular}{|c|c|c|c|c|c|c|c|c|}
\hline \multirow{2}{*}{ Categorias (cabeças) } & \multicolumn{2}{|c|}{ CriaCRUZA } & \multicolumn{2}{|c|}{ CriaZEBU } & \multicolumn{2}{|c|}{ CompCRUZA } & \multicolumn{2}{|c|}{ CompZEBU } \\
\hline & Valor & DP & Valor & $\mathrm{DP}$ & Valor & $\mathrm{DP}$ & Valor & $\mathrm{DP}$ \\
\hline Bezerros mamando & 1.107 & (59) & 1.039 & (26) & 1.094 & (46) & 931 & $(38)$ \\
\hline Bezerras mamando & 1.107 & (59) & 1.039 & (26) & 1.094 & (46) & 931 & (38) \\
\hline Fêmeas 1 ano & 1.043 & $(65)$ & 1.047 & (38) & 1.038 & (61) & 941 & (60) \\
\hline Fêmeas 2 anos & 849 & $(75)$ & 894 & (47) & 827 & (68) & 788 & (56) \\
\hline Fêmeas 3 anos & 711 & $(51)$ & 914 & (43) & 704 & (46) & 811 & (71) \\
\hline Fêmeas 4 anos & 460 & (44) & 755 & (64) & 457 & (42) & 675 & (73) \\
\hline Fêmeas 5 anos & 393 & $(35)$ & 500 & (49) & 393 & $(35)$ & 451 & $(48)$ \\
\hline Fêmeas 6 anos & 343 & $(38)$ & 437 & (44) & 343 & $(38)$ & 397 & (52) \\
\hline Garrotes 1 ano & 0 & $(0)$ & 0 & $(0)$ & 990 & (116) & 857 & (85) \\
\hline Bois de 2 anos & 0 & $(0)$ & 0 & $(0)$ & 0 & $(0)$ & 762 & $(217)$ \\
\hline Total & 6.012 & (35) & 6.625 & (28) & 6.939 & (41) & 7.544 & (61) \\
\hline
\end{tabular}

Esses valores são inferiores aos utilizados por Corrêa et al. (2000), que utilizaram um simulador para definir parâmetros para posterior implantação de um sistema físico de produção no Centro Nacional de Pesquisa de Gado de Corte Embrapa. Foi utilizada a capacidade de suporte de 1,4 UA/ha na seca. Segundo os autores, é exequível do ponto de vista técnico e econômico, bastando utilizar adubação de manutenção a cada três anos, conforme citado e recomendado por Macedo et al. (1993). A opção nesta simulação foi a de utilizar a capacidade de lotação média obtida na propriedade nos últimos cinco anos nas áreas com adequado manejo.

O rebanho ficou estabilizado em um número de UA's semelhantes. Porém, há grande diferença no número total de animais, de fêmeas em reprodução, de animais vendidos e de machos vendidos no ano (Tab. 1). Essas diferenças ocorrem pelo fato de os animais zebuínos terem um peso vivo em categoria inferior aos animais cruzados, resultando em sistemas com número distinto de animais. Segundo Borges et al. (2010), esse resultado pode ser associado à expressão máxima de heterose em cruzamentos de animais Bos taurus x Bos indicus.

As taxas de desfrute e a quantidade de peso vivo vendida/ha nos sistemas com animais cruzados foram $15,3 \%$ e $25,4 \%$ maiores aos exclusivamente zebuínos, respectivamente (Tab. 2). Isso se deve à maior precocidade dos cruzamentos com os taurinos e antecipação da idade ao abate e à reprodução. Para Barbosa (1999), precoce é o animal que chega mais cedo à idade adulta ou, em outras palavras, é aquele cujo esqueleto se completa precocemente, antes da idade comum à sua espécie. Tal acabamento se dá pela ossificação da zona de crescimento, dos ossos longos, e assim o animal para de crescer, com outra consequência importante: adquire a dentição definitiva também mais cedo. A maior precocidade também foi observada por Borges et al. (2010), que obtiveram uma idade de abate 82 dias mais cedo que os puros zebuínos, representando $11,3 \%$ de superioridade. As fêmeas cruzadas possuem maior peso corporal; porém, devido à antecipação da puberdade e do abate, há a liberação de mais áreas de pastagens. Esse fato faz com que o número de fêmeas em reprodução seja maior. A média nacional da taxa de desfrute, segundo a Associação Brasileira das Indústrias Exportadoras de Carnes (ABIEC, 2012), foi de $18,9 \%$ em 2011, inferior aos valores encontrados, tanto de cruzados quanto de zebuínos, e também inferior às taxas de desfrute obtidas em sistemas mais avançados tecnologicamente, como no Centro Nacional de Pesquisa de Gado de Corte da Embrapa, que foi de $32 \%$ (Corrêa et al., 2000).

Os sistemas CriaCRUZA e CompCRUZA obtiveram peso de bezerro desmamado por vaca $13,8 \%$ e $14 \%$ maior, respectivamente, quando comparado aos do zebuíno (Tab. 2), devido ao maior peso à desmama dos animais cruzados proveniente do efeito da heterose. Em um estudo com 882 animais da raça Tabapuã e cruzados com outras raças europeias, Borges et al. (2010) concluíram que os animais cruzados pesaram $27,69 \mathrm{~kg}$ a mais para peso a desmama aos 205 dias. Desempenho superior dos animais cruzados em relação aos zebuínos também foi observado 
por Perotto et al. (2001), que concluíram que o efeito fixo de grupo genético indicou melhor ganho de peso diário pós-desmama para o grupo Red Angus x Nelore (366g/dia), seguido de Marchigiana x Nelore (352 g/dia) e Guzerá x Nelore $(287 \mathrm{~g} / \mathrm{dia})$, indicativo de que cruzamentos de raças de corte com matrizes Nelore são opções disponíveis para os criadores para melhorar o desempenho de seus rebanhos. Os menores ganhos foram para os animas da raça Nelore (247g/dia).

Tabela 2. Indicadores de eficiência biológica dos sistemas simulados, CriaCRUZA, CompCRUZA, CriaZEBU, CompZebu

\begin{tabular}{lllll}
\hline Categorias & Cria & Cria & Comp & Comp \\
& CRUZA & ZEBU & CRUZA & ZEBU \\
\hline Total de animais ${ }^{1}$ (cabeças) & 5.024 & 5.624 & 5.483 & 6.309 \\
Fêmeas em reprodução (cabeças) & 2.708 & 2.582 & 2.695 & 2.346 \\
Taxa de natalidade média (\%) & 80 & 80 & 80 & 80 \\
Bezerros(as) desmamados(as) (cabeças) & 2.036 & 1.932 & 2.026 & 1.744 \\
Kg de bezerros(as) & 145,1 & 127,5 & 145,1 & 127,3 \\
desmamados(as)/vaca (cabeças) & & & & \\
Total de animais vendidos (cabeças) & 2.000 & 2.002 & 1.991 & 1.726 \\
Total de machos vendidos (cabeças) & 1.016 & 965 & 990 & 762 \\
Desfrute anual $(\%)$ & 24,4 & 22,7 & 24,4 & 19,8 \\
Kg de peso vivo vendido/ha $(\mathrm{kg})$ & 211,6 & 175,8 & 230,3 & 176,4 \\
Kg carne (carcaça) vendida/ha (kg) & 49,9 & 45,5 & 118,1 & 94,2 \\
\hline
\end{tabular}

${ }^{\mathrm{I}}$ Média dos doze meses do ano.

Quando avaliadas as vendas de carcaça/ha, os sistemas cruzados de cria e completo cruzados foram $9,6 \%$ e $25,4 \%$ maiores, em relação aos zebuínos, respectivamente (Tab. 2), graças à precocidade para a terminação e reprodução, aumentando assim a produtividade. Da mesma forma ocorre maior venda do sistema de ciclo completo quando comparado aos sistemas de cria. Barbosa et al. (2010), avaliando diferentes taxas de natalidade de um sistema de ciclo completo com rebanho misto zebu e cruzado, encontraram valores inferiores ao deste estudo, estando entre 141 e $149 \mathrm{~kg}$ peso vivo total vendido/ha/ano.

Quando comparados à estrutura de custos dos quatro sistemas (Tab. 3), observamos que os gastos relativos à manutenção de pastagens foram semelhantes, uma vez que é a mesma área em todos os sistemas simulados. Os gastos com instalações, benfeitorias, máquinas e equipamentos foram $15,4 \%$ maior para os sistemas de ciclo completo. Mesmo a propriedade tendo toda a estrutura de confinamento, os valores investidos e a sua manutenção foram retirados da simulação nos sistemas de cria, uma vez que não era necessário ao sistema. Para nenhum dos cenários simulados foram necessárias modificações (novos investimentos) nesses itens. Os custos fixos diferiram também no tocante à depreciação e juros relativos aos bovinos. Essa diferença é proporcional ao tamanho (cabeças) e peso ( $\mathrm{kg}$ ou UA) de cada rebanho.

O percentual do custo fixo, em relação ao custo total, foi $41,8 \%$ maior nos sistemas de cria quando comparado aos de ciclo completo, sendo $28,1 \%, \quad 27,1 \%$ e $18,8 \%, \quad 20,1 \%$ para CriaCRUZA, CriaZEBU, CompCRUZA e CompZEBU, respectivamente. Isso se deve à menor produtividade e menor número de cabeças do CriaCRUZA e CriaZEBU. Quando comparado ao sistema de cria, o cruzado tem um custo $9,5 \%$ maior que o zebu, diferente do constatado por Guimarães et al. (2006), em que o zebu teve um custo total $0,2 \%$ menor. Essa diferença é explicada em sua maior parte pela utilização da suplementação alimentar para os bezerros cruzados em aleitamento. $\mathrm{O}$ "creepfeeding" teve um custo anual de R\$ $71.455,87$, elevando o custo total em $4,6 \%$. 
Desempenho bioeconômico...

Tabela 3. Estrutura de custos dos sistemas simulados no $10^{\circ}$ ano de projeção dos sistemas simulados, CriaCRUZA, CompCRUZA, CriaZEBU, CompZebu

\begin{tabular}{|c|c|c|c|c|c|c|c|c|}
\hline \multicolumn{3}{|c|}{ CriaCRUZA } & \multicolumn{2}{|l|}{ CriaZEBU } & \multicolumn{2}{|c|}{ CompCRUZA } & \multicolumn{2}{|l|}{ CompZEBU } \\
\hline Categorias & Valor R\$ & $\%$ & Valor $\mathrm{R} \$$ & $\%$ & Valor $\mathrm{R} \$$ & $\%$ & Valor R\$ & $\%$ \\
\hline A.Custo fixo & $432.802,10$ & $(28,1)$ & $382.536,80$ & $(27,1)$ & $443.430,20$ & $(18,8)$ & $369.796,00$ & $(20,1)$ \\
\hline \multicolumn{9}{|c|}{ A.1.Rebanho bovino e animais de trabalho } \\
\hline Depreciação & $104.514,10$ & $(6,8)$ & $86.536,55$ & $(6,1)$ & $101.817,40$ & $(4,3)$ & $76.648,91$ & $(4,2)$ \\
\hline Juros & $174.897,20$ & $(11,3)$ & $142.609,40$ & $(10,1)$ & $169.734,70$ & $(7,2)$ & $121.269,00$ & $(6,6)$ \\
\hline \multicolumn{9}{|c|}{ A.2.Instalações e benfeitorias } \\
\hline Depreciação & $59.030,48$ & $(3,8)$ & $59.030,48$ & $(4,2)$ & $66.773,65$ & $(2,8)$ & $66.773,65$ & $(3,6)$ \\
\hline Juros & $34.316,00$ & $(2,2)$ & $34.316,00$ & $(2,4)$ & $40.074,98$ & $(1,7)$ & $40.074,98$ & $(2,2)$ \\
\hline \multicolumn{9}{|c|}{ A.3.Máquinas, equipamentos e veículo } \\
\hline Depreciação & $53.537,52$ & $(3,5)$ & $53.537,52$ & $(3,8)$ & $58.121,52$ & $(2,5)$ & $58.121,52$ & $(3,2)$ \\
\hline Juros & $4.985,53$ & $(0,3)$ & $4.985,53$ & $(0,4)$ & $5.386,63$ & $(0,2)$ & $5.386,63$ & $(0,3)$ \\
\hline $\begin{array}{l}\text { A.4.Imposto } \\
\text { territorial }\end{array}$ & $1.521,30$ & $(0,1)$ & $1.521,30$ & $(0,1)$ & $1.521,30$ & $(0,1)$ & $1.521,30$ & $(0,1)$ \\
\hline $\begin{array}{l}\text { B.Custo } \\
\text { variável }\end{array}$ & $1.109 .694,00$ & $(71,9)$ & $1.026 .777,00$ & $(72,9)$ & $1.915 .539,00$ & $(81,2)$ & $1.466 .089,00$ & $(79,9)$ \\
\hline $\begin{array}{l}\text { B.1.Manutenção } \\
\text { de benfeitorias }\end{array}$ & $28.273,71$ & $(1,8)$ & $28.273,71$ & $(2,0)$ & $32.629,24$ & $(1,4)$ & $32.629,24$ & $(1,8)$ \\
\hline $\begin{array}{l}\text { B.2.Manut. máq. e } \\
\text { equipamentos }\end{array}$ & $19.216,55$ & $(1,3)$ & $19.216,55$ & $(1,4)$ & $20.935,55$ & $(0,9)$ & $20.935,55$ & $(1,1)$ \\
\hline $\begin{array}{l}\text { B.3.Combustível } \\
\text { de veículo }\end{array}$ & $4.032,00$ & $(0,3)$ & $4.032,00$ & $(0,3)$ & $4.032,00$ & $(0,2)$ & $4.032,00$ & $(0,2)$ \\
\hline $\begin{array}{l}\text { B.4.Controle } \\
\text { sanitário }\end{array}$ & $29.465,06$ & $(1,9)$ & $27.665,50$ & $(2,0)$ & $33.347,04$ & $(1,4)$ & $32.708,18$ & $(1,8)$ \\
\hline B.5.Sal mineral & $\begin{array}{l}109.430,9 \\
0\end{array}$ & $(7,1)$ & $96.701,59$ & $(6,9)$ & $106.574,20$ & $(4,5)$ & $100.573,70$ & $(5,5)$ \\
\hline $\begin{array}{l}\text { B.6.Alimentação } \\
\text { (confinamento) }\end{array}$ & 0,00 & $(0,0)$ & 0,00 & $(0,0)$ & $680.818,60$ & $(28,9)$ & $283.086,30$ & $(15,4)$ \\
\hline $\begin{array}{l}\text { B.7.Suplem. } \\
\text { alimentar em } \\
\text { pasto }\end{array}$ & $71.455,87$ & $(4,6)$ & 0,00 & $(0,0)$ & $68.646,23$ & $(2,9)$ & $39.686,77$ & $(2,2)$ \\
\hline $\begin{array}{l}\text { B.8.Mão de obra } \\
\text { e encargos }\end{array}$ & $\begin{array}{l}106.207,9 \\
0\end{array}$ & $(6,9)$ & $121.380,50$ & $(8,6)$ & $156.951,70$ & $(6,7)$ & $141.189,10$ & $(7,7)$ \\
\hline $\begin{array}{l}\text { B.9.Adubação } \\
\text { de pastagens }\end{array}$ & $\begin{array}{l}611.233,9 \\
0\end{array}$ & $(39,6)$ & $611.233,90$ & $(43,4)$ & $611.233,90$ & $(25,9)$ & $611.233,90$ & $(33,3)$ \\
\hline $\begin{array}{l}\text { B.10.Impostos } \\
\text { sobre vendas de } \\
\text { animais } \\
\text { (FUNRURAL) }\end{array}$ & $33.319,23$ & $(2,2)$ & $30.153,33$ & $(2,1)$ & $77.654,69$ & $(3,3)$ & $59.247,05$ & $(3,2)$ \\
\hline $\begin{array}{l}\text { B.11.Outras } \\
\text { despesas }\end{array}$ & $30.000,00$ & $(1,9)$ & $30.000,00$ & $(2,1)$ & $30.000,00$ & $(1,3)$ & $30.000,00$ & $(1,6)$ \\
\hline $\begin{array}{l}\text { B.12.Juros sobre } \\
\text { o capital }\end{array}$ & $67.059,03$ & $(4,4)$ & $58.119,77$ & $(4,1)$ & $92.715,90$ & $(3,9)$ & $110.767,10$ & $(6,0)$ \\
\hline $\begin{array}{ll}\begin{array}{l}\text { C.Custo total } \\
(A+B)\end{array} & 1 \\
\end{array}$ & $542.496,00$ & (100) & $1.409 .314,00$ & $(100)$ & $2.358 .969,00$ & $(100)$ & $1.835 .885,00$ & (100) \\
\hline
\end{tabular}

Os gastos relativos à alimentação, controle sanitário, mão de obra, impostos sobre a venda e juros referentes ao capital também foram proporcionais ao tamanho e peso de cada rebanho no sistema. Os valores dispendidos em confinamento somente foram considerados nos sistemas que adotaram essa tecnologia (CompCRUZA e CompZEBU).
Os custos com a manutenção de pastagens (irrigadas e de sequeiro) estão representados na Tabela 3, pelos custos com adubo e distribuição, e representaram de 25,9 a $42,6 \%$ do custo total, sendo percentualmente mais representativos nos sistemas de cria.

Os sistemas que adotaram o ciclo completo tiveram um custo total ( $\mathrm{R} \$ / \mathrm{ano}$ ) muito superior aos que adotaram a venda de bezerros, sendo 
52,9 e 30,3\% superiores aos de CriaCRUZA e CriaZEBU, respectivamente. Entre os grupos genéticos, ressalta-se a diferença, sendo que o CriaCRUZA teve um custo total (R\$/ano) 9,5\% maior e compCRUZA $28,5 \%$ superior. A produção de animais cruzados, quando comparada à de zebuínos ou ainda de ciclo completo comparada com cria, demanda maior aporte de recursos para fluxo de caixa.

A Tabela 4 mostra as receitas provenientes das vendas das diversas categorias nos quatro sistemas simulados com rebanho estabilizado, no $10^{\circ}$ ano. Os sistemas que adotaram o ciclo completo obtiveram uma receita total $30,2 \%$ maior. Dentre os sistemas, os que apresentaram as maiores receitas foram aqueles que apresentaram a maior quantidade de peso vivo vendido/ha (Tab. 4). Isso está relacionado com a maior eficiência de utilização de energia para ganho de peso, conforme relatado por Barbosa et al. (2010).

Tabela 4. Receitas com rebanho estabilizado, no $10^{\circ}$ ano de simulação, dos sistemas simulados, CriaCRUZA, CompCRUZA, CriaZEBU, CompZebu

\begin{tabular}{lcccc}
\hline Categorias & CriaCRUZA-R $\$$ & CriaZEBU-R $\$$ & CompCRUZA-R \$ & CompZEBU-R\$ \\
\hline Bezerro desmamado & $934.888,00$ & $766.621,00$ & - & - \\
Boi gordo cria & - & - & $1.960 .414,00$ & $1.493 .067,00$ \\
Vaca velha & $469.926,00$ & $526.580,00$ & $469.926,00$ & $402.915,00$ \\
Novilha descarte & $193.220,00$ & $133.397,00$ & $164.289,00$ & $114.973,00$ \\
Touro velho & $60.178,00$ & $49.432,00$ & $58.566,00$ & $43.522,00$ \\
Novilha vazia & $206.998,00$ & $169.292,00$ & $202.745,00$ & $145.603,00$ \\
Vaca 1 cria vazia & $408.857,00$ & $357.408,00$ & $381.936,00$ & $316.928,00$ \\
Vaca > 2 cria vazia & $302.703,00$ & $208.302,00$ & $302.703,00$ & $173.924,00$ \\
\hline Total & $2.576 .770,00$ & $2.211 .032,00$ & $3.540 .579,00$ & $2.690 .932,00$ \\
\hline
\end{tabular}

Os que utilizaram as raças zebuínas, apesar de terem uma venda de peso vivo vendido/ha semelhante, se diferem na receita, pois a quantidade de bovinos machos vendidos é 16,2\% maior no sistema com animais cruzados, resultando em uma venda com receita $24,8 \%$ superior. A receita total de CriaCRUZA foi próxima de CompZEBU, sendo assim atividades economicamente semelhantes (Tab. 4). A Tabela 5 mostra a média nos dez anos de projeção dos principais indicadores econômicos anuais.

Tabela 5. Indicadores econômicos anuais (média dos dez anos projetados) dos sistemas simulados, CriaCRUZA, CompCRUZA, CriaZEBU, CompZEBU

\begin{tabular}{lcccc}
\hline Categorias & CriaCRUZA & CriaZEBU & CompCRUZA & CompZEBU \\
\hline $\begin{array}{l}\text { Margem bruta (R\$) } \quad(= \\
\text { receitas totais - custos variáveis) }\end{array}$ & $1.329 .332,00$ & $1.159 .455,00$ & 1.571 .729 & $1.306 .749,00$ \\
Margem operacional (R\$) & & & & \\
(= lucro operacional) & $1.124 .920,00$ & $963.761,50$ & 1.355 .439 & $1.106 .549,00$ \\
Margem Operacional/ha (R\$) & 295,77 & 253,40 & 356,38 & 290,94 \\
Margem Operacional/cabeça (R\$) & 223,91 & 171,37 & 247,21 & 175,39 \\
Lucro total/ha (área total) (R\$) & 168,05 & 140,67 & 207,48 & 160,40 \\
Lucro total/cabeça vendida (R\$) & 426,11 & 356,33 & 528,45 & 471,26 \\
Lucro total (R) & $852.210,70$ & $713.371,70$ & $1.052 .137,0$ & $813.386,40$ \\
Taxa Interna de Retorno - TIR & $17,2 \%$ & $14,6 \%$ & $18,9 \%$ & $16,2 \%$ \\
Taxa de desconto & $6.75 \%$ & $6.75 \%$ & $6.75 \%$ & $6.75 \%$ \\
Valor Presente Líquido - VPL & $\mathrm{R} \$ 10.151 .896,54$ & $\mathrm{R} \$ 7.955 .230,38$ & $\mathrm{R} \$ 11.749 .329,42$ & $\mathrm{R} \$ 9.568 .293,51$ \\
\hline
\end{tabular}

Observa-se na Tabela 5 que os valores da margem bruta, margem operacional e lucro foram positivos e mostram a viabilidade econômica de qualquer um dos quatro sistemas adotados. Do ponto de vista econômico, o mais vantajoso seria o CompCRUZA, sendo superior em relação ao parâmetro lucro em 29,4\%, 23,5\% e $47,5 \%$ quando comparados com o CompZEBU, 
CriaCRUZA e CriaZEBU, respectivamente. O lucro e margem bruta do CompZEBU e do CriaCRUZA foram semelhantes, sendo o lucro do cruzado superior em 4,8\%. De acordo com Nix (1995), a comparação da margem bruta pode ser uma forma útil de se avaliar a eficiência técnica dos sistemas de produção, principalmente quando os recursos utilizados nos sistemas são semelhantes.

Nos sistemas que adotam a venda de bezerros, o CriaCRUZA obteve um lucro $19,5 \%$ maior que o CriaZEBU, resultado semelhante aos encontrados por Guimarães et al. (2006), que foram $24 \%$ superior quando comparados Nelore e Nelore X Angus. Nos sistemas que adotam ciclo completo, o lucro do CompCRUZA foi $29,4 \%$ maior que o CompZEBU. Nota-se a superioridade em todos os parâmetros econômicos encontrados nos sistemas que adotam o cruzamento de raças zebuínas com taurinas em relação aos sistemas de raças zebuínas somente (Tab. 5). Pode-se observar que os quatro sistemas apresentaram índices econômicos e financeiros positivos se comparados aos rendimentos da caderneta de poupança (6\% ao ano) e aos praticados no financiamento agropecuário brasileiro $(6,75 \%$ ao ano). A TIR e o VPL, quando considerada a venda do patrimônio no final dos dez anos, foi superior para os sistemas que adotam o cruzamento de zebuínos com raças europeias (CriaCRUZA e CompCRUZA). Entre os sistemas de criação que adotam o ciclo completo (CompCRUZA e CompZEBU), também foi melhor remunerado o que se utiliza do cruzamento. Os resultados de fluxo de caixa pela TIR encontrados por Barbosa et al. (2008), com o preço de terra incluído no cálculo, indicaram uma TIR de 13,26\% para ciclo completo em propriedades de Minas Gerais, e 8,61\%, para propriedades na Bahia. O sistema CriaCRUZA foi mais rentável em 4,8\% que o CompZEBU. Outros autores também encontraram valores positivos para VPL em sistemas de produção de bovinos de corte. Abreu et al. (2003), simulando 5 cenários na implantação de período de monta em sistema de cria, encontraram o VPL variando de $\mathrm{R} \$ 512.866,00$ a $\mathrm{R} \$$ 668.743,00 (com uma taxa de desconto de $10 \%$ ao ano), e Guimarães et al. (2006), simulando a produção de bezerros da raça Nelore, encontraram o VPL de R\$ 423.100,27 (US\$ 233.757,00).

\section{CONCLUSÕES}

Os quatro sistemas intensivos simulados apresentaram viabilidade econômica, obtendo rentabilidade superior aos juros de poupança ou de financiamento agropecuário. Os sistemas que utilizaram os cruzamentos de zebuínos $\mathrm{x}$ taurinos tiveram um resultado econômico superior quando comparados aos que utilizaram somente as raças zebuínas, devido à maior produtividade (taxa de desfrute e $\mathrm{kg}$ de peso vendido/ha) e, consequentemente, maior lucro. Quando comparados, dentro do mesmo grupo genético, os sistemas de produção de ciclo completo foram mais produtivos e rentáveis que os de cria, devido à maior venda de $\mathrm{kg}$ de peso vivo e de carcaça/ha.

Devido ao maior peso vivo dos animais cruzados, a quantidade de animais em um rebanho estabilizado é menor, porém a quantidade vendida em $\mathrm{kg}$ de carcaça e peso vivo/ha e a taxa de desfrute são maiores em relação ao sistema com zebuínos, em decorrência da maior precocidade da idade ao primeiro parto e ao abate dos animais cruzados.

\section{REFERÊNCIAS}

ABIEC. [2012]. Pecuária Brasileira. Disponível em: <http://www.abiec.com.br/3_pecuaria.asp>. Acessado em: 1 fev. 2012.

ABREU, U.G.P.D.; CEZAR, I.M.; TORRES, R.D.A. Análise bioeconômica da introdução de período de monta em sistemas de produção de rebanhos de cria na região do Brasil Central. Rev. Bras. Zootec., v.32, p.1198-1206, 2003.

BARBOSA, F.A.; GRAÇA, D.S.; ANDRADE, V.J.E.A. Viabilidade econômica da terminação de bovinos de corte em sistema intensivo de pastagem e confinamento. In: REUNIÃO ANUAL DA SOCIEDADE BRASILEIRA DE ZOOTECNIA, 45., 2008, Lavras. Anais... Lavras: SBZ, 2008.

BARBOSA, F.A.; GRAÇA, D.S.; ANDRADE V.J. et al. Produtividade e eficiência econômica de sistemas de produção de cria, recria e engorda de bovinos de corte na região sul do estado da Bahia. Arq. Bras. Med. Vet. Zootec., v.62, p.677$685,2010$. 
BARBOSA, P.F. Raças e estratégicas de cruzamento para produção de novilhos precoces. In: SIMPÓSIO DE PRODUÇÃO DE GADO DE CORTE, 1., 1999, Viçosa. Anais... Viçosa: UFV, 1999.

BORGES, D.N.; BARBOSA, F.; CABRAL FILHO, S.L.S. et al. Desempenho produtivo de bovinos tabapuã e seus cruzados em pastagens de braquiárias no estado da bahia. In: CONGRESSO BRASILEIRO DE ZOOTECNIA, 20, 2010, Palmas. Anais... Palmas: Zootec, 2010. (CDROM)

CARVALHO, L.C.S.; ELIA, B.S.; DECOTELLI, C.A. Matemática Financeira Aplicada. 1. ed. [S.1.]: FGV, 2009. 160 p.

CEZAR, I.M. Modelo bioeconômico de produção de bovino de corte. I. Descrição do modelo. Pesq. Agropec. Bras., v.17, p.941-949, 1982.

CORRÊA, E.S.; VIEIRA, A.; COSTA, F.P.E.A. Sistema semi-intensivo de produção de carne de bovinos nelores no Centro-Oeste do Brasil. Campo Grande: Embrapa/CNPGC, 2000. (Documento, 95).

COSTA, F.P.; PACHECO, J.A.C.; CORRÊA, E.S.E.A. Estimativa do custo de produção da carne bovina para a região centro-oeste. Campo Grande: Embrapa/CNPGC, 1986. (Comunicado Técnico, 30).
FERREIRA, G.; CARDOZO, O.; LIMA, J.M.S. Modelo bio-economico para toma de decisiones en engorde de novillos a pastoreo. In: EVERLING, D.M.; QUADROS, F.L.F.; VIÉGAS, J. et al. Modelos para tomada de decisões na produção de bovinos e ovinos. Santa Maria, Pallotti, 2002. p.121-145.

FRANK, R.G. Introducción al cálculo de costos agropecuarios. Buenos Aires: El Ateneo, 1978, $37 \mathrm{p}$.

GUIMARÃES, P.H.S.; MADALENA, F.E.; CEZAR, I.M. Comparative economics of Holstein/Gir F1 dairy female production and conventional beef cattle suckler herds - A simulation study. Agricult. Systems, v.88, p.111124, 2006.

NIX, J. Farm management pocketbook. Kent: Wye College, 1995. 220 p.

PEROTTO, D.; CUBAS, A.C.; ABRAHÃO, J.J.S. et al. Ganho de Peso da Desmama aos 12 Meses e Peso aos 12 Meses de Bovinos Nelore e Cruzas com Nelore. Rev. Bras. Zootec., v.30, p.730-735, 2001.

TANURE, S.; NABINGER, C. Ferramentas de gerenciamento bioeconômico e suporte à decisão em empresas de pecuária de corte. In: CONGRESO INTERNACIONAL DE LA CARNE BOVINA, 4., 2010, Asunción. Anais... Asunción: [s.n.]. 2010. Disponível em: <http://www.agr.una.py/congreso/imagen/presen taciones/Soraya_Tanure/Palestra1.pdf $>$. Acessado em: 28 ago. 2011. 